\title{
sciendo
}

RESEARCH PAPERS FACULTY OF MATERIALS

SCIENCE AND TECHNOLOGY IN TRNAVA

SLOVAK UNIVERSITY OF TECHNOLOGY

IN BRATISLAVA

2018, Volume 26, Number 43

DOI $10.2478 /$ rput-2018-0026

\section{ON THE PROBLEM OF THE ION ENERGY LOSS IN THIN ABSORBERS}

\author{
Stanislav MINÁRIK, Róbert RIEDLMAJER \\ SLOVAK UNIVERSITY OF TECHNOLOGY IN BRATISLAVA, \\ FACULTY OF MATERIALS SCIENCE AND TECHNOLOGY IN TRNAVA, \\ ADVANCED TECHNOLOGIES RESEARCH INSTITUTE, \\ ULICA JÁNA BOTTU 2781/25, 91724 TRNAVA, SLOVAK REPUBLIC \\ e-mail: stanislav.minarik@stuba.sk, robert.riedlmajer@stuba.sk \\ Received: 26.04.2018, Accepted: 10.01.2019, Published: 29.01.2019
}

\begin{abstract}
Exact determination of energy loss of ion in materials is still a non-trivial task because of relatively complicated changes of the ion 's stopping power $S(E)$ during transport of the probing ion inside the material structure. Energy loss of ion in the material structure always depends on the current value of continually decreasing energy of the ion, indeed. A purely theoretical approach can be applied to the energy loss calculation in some typical cases. Average energy loss of ion can be determined by means of the Bethe-Bloch analytical theory (1-6) (in high velocity region) and the Lindhard-Scharff-Schiøtt (LSS) theory (7-10) (in low velocity region). Currently, there is no acceptable exact theory to determine the energy loss of ion in intermediate-velocity region (11) and experimental data must be used in that case. Basically, only a finite number of discrete experimental data is always available. Therefore, if the problem how much energy the projectile ion loses in a certain distance travelled is solved, the modelling of function $S(E)$ based on some acceptable assumptions must be applied.

In this contribution, we present the energy loss calculations in intermediate-velocity region of ion based on linear interpolation of experimental data. Calculation was carried out for a kapton foil using the data taken from Ziegler, Biersack (12). Energy values of an ion along its trajectory inside the foil were found, and the mean projected range of ion penetrating into the foil was calculated. Finally, the energy resolution was evaluated taking into account straggling provided that the foil is used as an absorber in the ERDA experiment.
\end{abstract}

\section{Key words}

Nuclear stopping power, electronic stopping power, Bethe-Bloch theory, LSS theory, mean projected range, linear interpolation 


\section{INTRODUCTION}

If the mean value of stopping power $S$ is known, the mean distance travelled by ion in media can be determined by:

$$
x(E)=\int_{E_{0}}^{E} \frac{d \varepsilon}{S(\varepsilon)},
$$

where $E_{0}$ is initial energy and $E$ is current energy of the ion. Formula [1.1] is essential for the energy loss evaluation. However, the main problem is to find the analytical form of the function $S(E)$ which we need to integrate.

Slowing down the force (stopping power) $S(E)$ of ions in a matter is a subject which still wins a great theoretical and experimental interest. The stopping power is the mean decelerating force applied to the ion travelling in the material. When a swift ion strikes the target, the transfer of its energy to the target material structure begins immediately. Two basic energy transfer mechanisms are dominant in such a case. One arises from collisions of ion with target atoms (nuclear stopping power $S_{n}$ ) and the other from processes of excitation and ionisation of the target electrons (electronic stopping power $S_{e}$ ). The total stopping power is determined by the sum of the above-mentioned components (13):

$$
S_{\text {total }}=S_{n}+S_{e}=\left(\frac{d E}{d x}\right)_{n}+\left(\frac{d E}{d x}\right)_{e} .
$$

It was found that the ions moving fast lose kinetic energy mainly via electronic stopping (14). If the ion moves slowly, it carries all of its electrons with it. If the ion is moves faster than the fastest electrons in the target, it loses all its electrons and is completely ionized. When the ion velocity is between the two cases, the ion is partially stripped, which leads to a considerably more complicated description (15), because the ion may lose electrons to, and capture them from, the target. So the values of velocity of the ion can be classified under one of the following three regions:

1) Low-velocity region, where the ion velocity $v$ is below the Bohr velocity $v_{0}$ of the target electrons, i.e. the velocity of fastest electrons in the material (in the electron gas theory, the limit is commonly the Fermi velocity). The ion moving at this velocity is called slow and average electronic stopping power of such an ion results from the Lindhard-Scharff-Schiøtt (LSS) theory (7-10):

$$
\left\langle\frac{d E}{d x}\right\rangle_{e}=-\frac{32 \pi^{2} \varepsilon_{0} \hbar^{3}}{m_{e} e^{2}} \frac{\rho}{m_{a t}} z^{\frac{7}{6}} Z\left(z^{\frac{2}{3}}+Z^{\frac{2}{3}}\right)^{-\frac{3}{2}} v .
$$

The universal reduced nuclear stopping power of ion in this low-velocity region was determined by G. Moliere in the framework of the Thomas-Fermi potential (16). The following empirical formula can used to approximate the nuclear stopping power calculation near the maximum of $S_{n}(E)$ :

$$
\left\langle\frac{d E}{d x}\right\rangle_{n}=-\frac{\rho \pi e^{2} a}{8 \varepsilon_{0} m_{a t}}\left(\frac{z Z M_{i o n}}{m_{a t}+M_{i o n}}\right),
$$

where $\rho$ is density of the material, $e$ is electron charge, $\varepsilon_{0}$ is electric permittivity of vacuum, $z$ is charge state of ion, $Z$ is atomic number of target atoms, $M_{\text {ion }}$ is mass of ion $m_{a t}$ is mass of target atoms, $\hbar$ is the reduced Planck constant and parameter $a=1,4.10^{-2} \mathrm{~nm}$. The nuclear stopping power is of the same order of magnitude as the electronic stopping power for very slow ions and must be taken into account. 
2) High-velocity region, where the ion velocity $v>v_{m} . v_{m}$ is the mean velocity of electrons filling the levels of a neutral atom with nuclear charge $z$ obtained from the Thomas-Fermi statistical theory (17):

$$
v_{m}=v_{0} z^{\frac{2}{3}}
$$

The ion moving at this velocity is called swift and the average electronic stopping power of such an ion results from the Bethe-Bloch theory (1-6):

$$
\left\langle\frac{d E}{d x}\right\rangle_{e}=-\frac{4 \pi z^{2}}{m_{e} v^{2}} \frac{N_{A} Z \rho}{A M_{u}}\left(\frac{e^{2}}{4 \pi \varepsilon_{0}}\right)^{2}\left[\ln \left(\frac{2 m_{e}}{I} v^{2}\right)\right],
$$

where $A$ its relative atomic mass of target atoms, $N_{A}$ the Avogadro number, $M_{u}$ the molar mass constant and $m_{e}$ is electron mass. In the Bethe theory, the atoms of material are characterized by means of excitation potential $I$. The effect of nuclear stopping power is very small for a swift ion and can be neglected in the high-velocity region.

3) Intermediate-velocity region, which is the intermediate area between the low and high velocities:

$$
v_{0}<v<v_{m}
$$

There is no acceptable formula to calculate energy loss of ion in intermediate-velocity range and we have only experimental data. In general, it can be concluded that velocity proportional energy loss has been in fact observed experimentally for most of common materials.

Our research is oriented just on the evaluation of distribution of the mean energy value of the ion along its trajectory in material. The current contribution deals with the problem of the SRIM data applicability in estimating the mean projected range of ion. Below, we discuss the effect of the straggling on the energy resolution when a thin absorber is used.

\section{MATHEMATICAL TREATMENT}

\section{Linear interpolation of SRIM data}

We start from a set of points $\left[E_{i}, S_{i}\right], i=1,2, \ldots N$, where $E_{i}$ are values of energy and $S_{i}$ represents the corresponding stopping power downloaded from SRIM (see the points in the graph shown in Fig.1). The $N$ points make $(N-1)$ intervals between them. It is necessary to

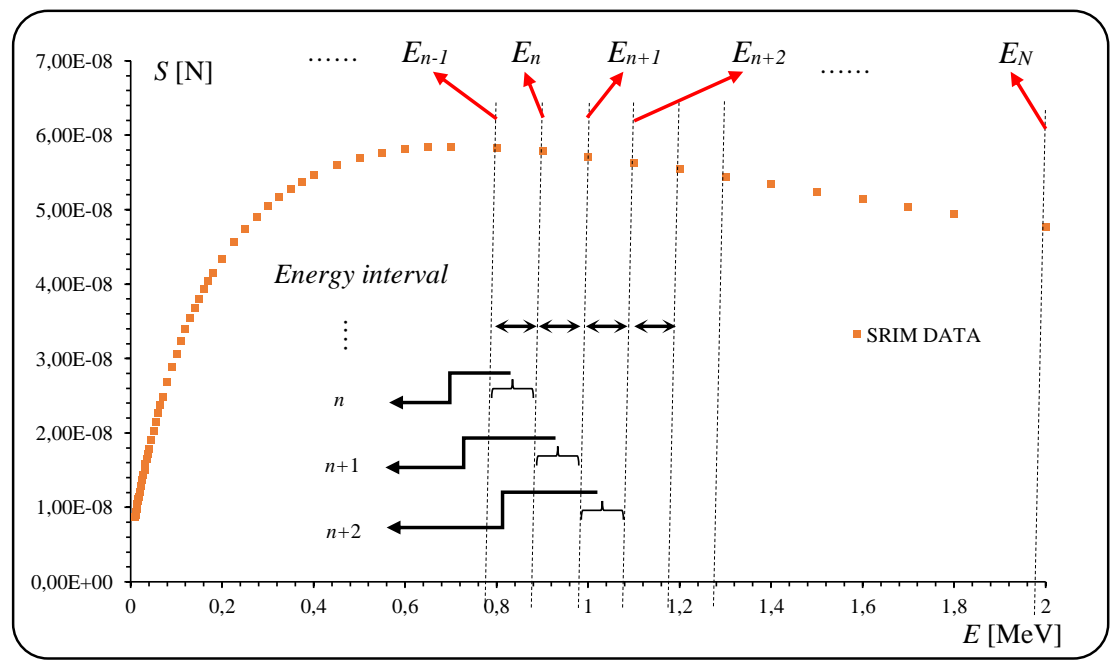

Fig. 1 A scheme illustrating the interpolation of SRIM data 
know the function $S=S(E)$ to apply the formula [1.1] for the ion range determination. This function can be approximated using linear interpolation at which a piecewise continuous line passing through each of the points is found. The two-point boundary value problem must be solved in this procedure. In this section, we briefly describe the procedure for determination of this line in the form of a separate linear polynomial for each interval (each with its own coefficients).

We look for the linear polynomial in the following form:

$$
S^{(n)}(E)=a_{1}^{(n) E}+a_{0}^{(n)} \quad \cdots \quad \text { for } n=2,3,4 .
$$

It can be considered that in the first interval (for $n=1$, i.e. at the lowest energies of ion), the mentioned function $S(E)$ follows directly from the LSS theory and it takes a form:

$$
S^{(1)}(E)=C \sqrt{E},
$$

where $C$ is constant.

It is necessary to determine the coefficients $a_{0}{ }^{(n)}, a_{1}{ }^{(n)}$ for polynomials [2.1.1] in all $N$-1 energy intervals (except the first interval). For this purpose, we need $2(N-1)$ independent equations. The coefficients of the polynomials [2.1.1] must be determined in such a way that the function $S(E)$ is continuous in all points $\left[E_{i}, S_{i}\right]$. Therefore, if we use designations:

$$
S^{(n)}\left(E_{n-1}\right)=S_{n-1} \quad, S^{(n)}\left(E_{n}\right)=S_{n},
$$

the following conditions must apply for each point $n=2,3,4, \ldots . N$ :

$$
\begin{aligned}
& a_{1}^{(n)} E_{n-1}+a_{0}^{(n)}=S_{n-1} \\
& a_{1}^{(n)} E_{n}+a_{0}^{(n)}=S_{n}
\end{aligned}
$$

For the first point $(n=1)$, i.e. at the lowest measured value of energy $E_{1}$, the following formulas can be written:

$$
S^{(1)}\left(E_{1}\right)=S_{1}=C \sqrt{E_{1}}
$$

Formulas for any coefficients $a_{0}^{(n)}, a_{1}{ }^{(n)}$ can be easily found from the system of equations (2.1.4), (2.1.5):

$$
\begin{aligned}
& a_{0}^{(n)}=\frac{E_{n} S_{n-1}-E_{n-1} S_{n}}{E_{n}-E_{n-1}}, \\
& a_{1}^{(n)}=\frac{S_{n}-S_{n-1}}{E_{n}-E_{n-1}} .
\end{aligned}
$$

Applying the results [2.1.7] and [2.1.8] in polynomials [2.1.1] and considering [2.1.2], we get interpolation function $S(E)$ in the whole required energy interval. For example, the graph of this function in the energy interval from 0 up to $2.2 \mathrm{MeV}$ for $\mathrm{He}^{2+}$ ion in the kapton foil is shown in Fig.2. 


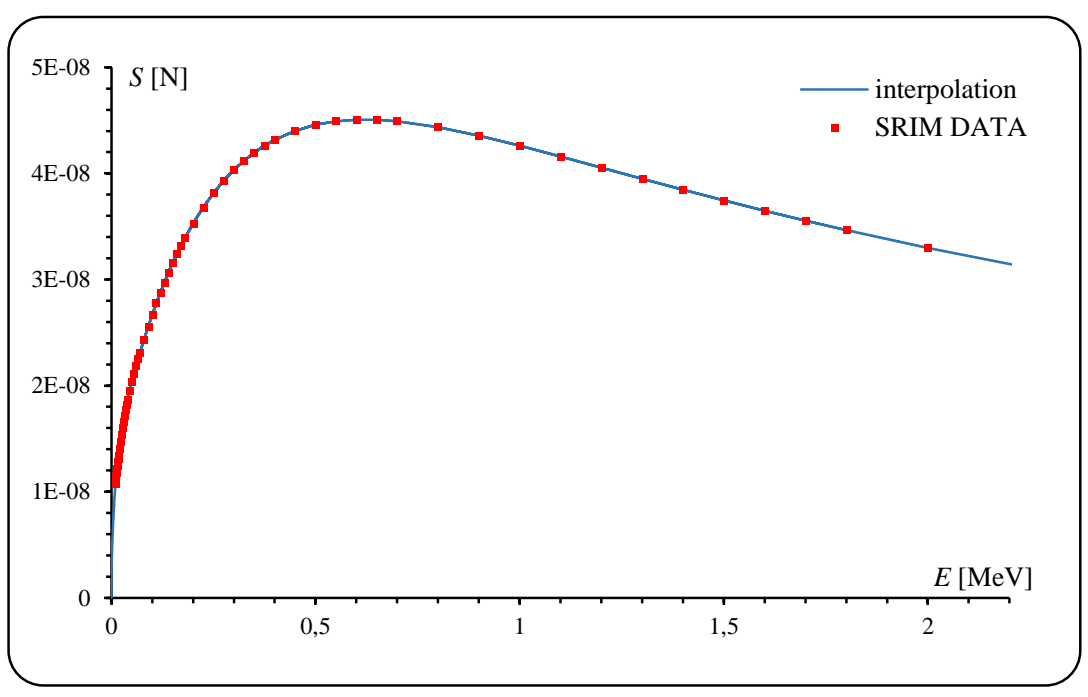

Fig. 2 Piecewise continuous curve as a result of the linear interpolation

Values of the stopping power for individual constituent in this kapton foil were taken from SRIM code. Next, the procedure described above was applied for the determination of the stopping power values in the kapton structure. This function can be used to determine the ion range penetration into material.

\section{Mean projected range of ion penetrating into solids}

The energy loss process of ions has been widely investigated in various media by a variety of methods. Understanding the process is an imperative in many areas of materials science, both in application and basic research. It is also very important in using practical analytical techniques, such as ion beam analysis.

The energy losses of ion during transport in a material structure can be calculated using formula [1.1] presented in Section 1. The stopping power of ions in a matter $S$ is usually given for different energies of ion. If the function $S(E)$ can be evaluated in the form [2.1.1], for any interval $<E_{n-1}, E_{n}>$ we have:

$$
S^{(n)}(E)=-\frac{d E^{(n)}}{d x}=a_{1}^{(n)} E+a_{0}^{(n)} . \quad(n=2,3,4, \ldots N)
$$

The following equation follows from [2.2.1]:

$$
\int \frac{d E^{(n)}}{a_{1}^{(n)} E+a_{0}^{(n)}}=-\int d x
$$

Solutions to the equation [2.2.2] can be found in all energy intervals. This solution can be written in the form:

$$
x^{(n-1)}=x_{k}^{(n)}+\frac{1}{a_{1}^{(n-1)}} \ln \left|\frac{a_{1}^{(n-1)} E_{k}^{(n-1)}+a_{0}^{(n-1)}}{a_{1}^{(n-1)} E^{(n-1)}+a_{0}^{(n-1)}}\right|
$$

where $x_{k}^{(n)}=0$. It should be noted that experimental values $E_{i}$ and $S_{i}$ (for $i=N, \ldots 2$ ) represent boundary conditions to the solution of equation [2.2.1], and different types of solution must be applied in each of the intervals. The distance travelled by ion in material structure must be determined step by step. Calculation must begin with $n=N$; then $n$ gradually decreases by step 
1 and the values $x_{n+1}$ always result from the calculation with previous $n$ (i.e. from the calculation in the previous energy interval). In any case, we must consider $x_{N+1}=0$ and $E_{N}=E_{0}$ in [2.2.3] for $n=N$ because the travelled distance is equal to zero at the moment when the ion with energy $E_{0}$ is entering into the material ( $E_{0}$ is initial energy of ion). Finally, in the first interval (i.e. for $n=1$ ), the equation to be solved takes the form [2.1.2], and the next solution can be written at the lowest energies of ion:

$$
x=x_{2}+\frac{2}{C}\left(\sqrt{E_{1}}-\sqrt{E}\right), \text { where } C=\frac{S_{1}}{\sqrt{E_{1}}} .
$$

Bragg curve of ion in the material structure can be calculated using the algorithm based on the presented results (for example see Fig.3).

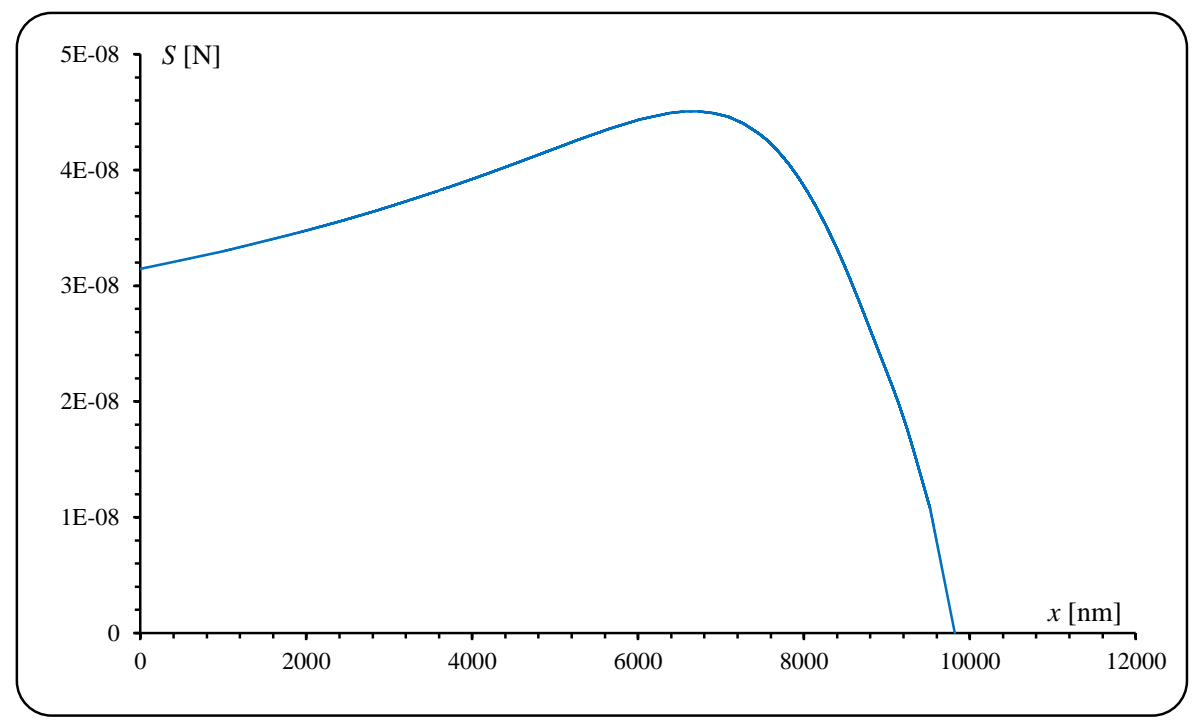

Fig. 3 Calculated Bragg curve for $2.2 \mathrm{MeV} \mathrm{He} e^{2+}$ ion in the kapton foil

The importance of formulas [2.2.3] is clear; $E$ is the energy of ion after travelling a distance $x$ in material. The results enable the calculation of energy loss of ion which is slowing down in a material structure (see Fig.4). The above-mentioned formulation for the stopping power calculation of $\mathrm{He}^{2+}$ ion in the kapton foil was used in intermediate-velocity region where we can rely only on the SRIM database of discrete values of stopping power.

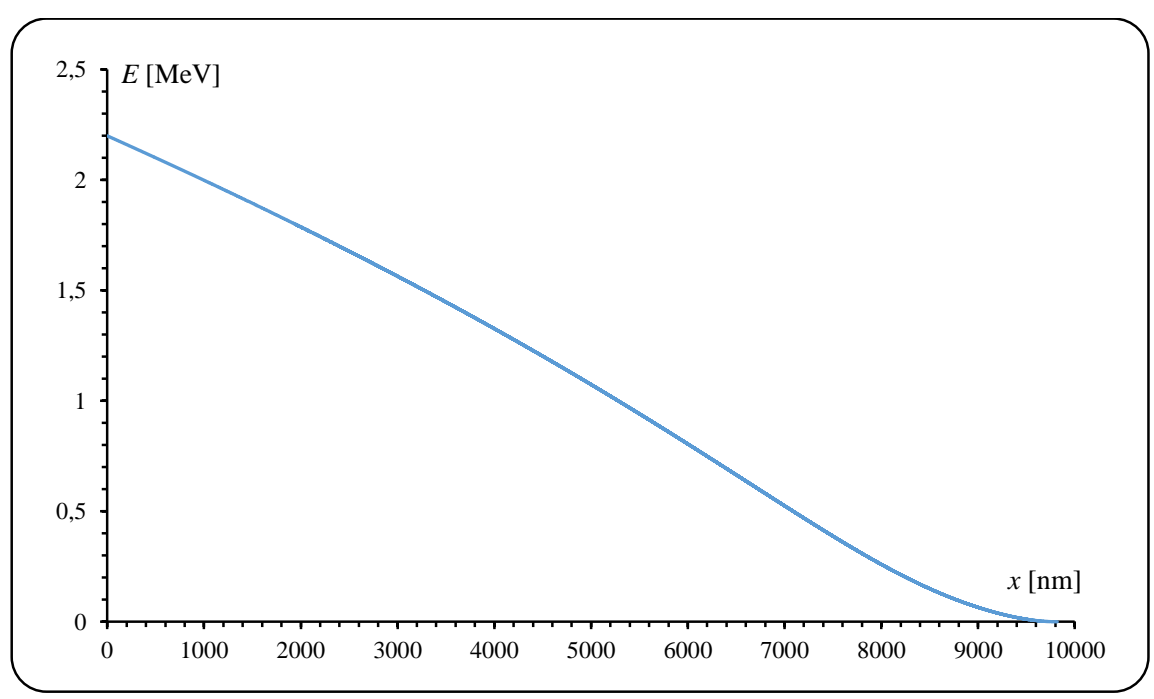

Fig. 4 Calculated energy decrease of $2.2 \mathrm{MeV} \mathrm{He}^{2+}$ ion penetrating in the kapton foil 


\section{Effect of the straggling}

In the previous sections, we only discussed the mean energy loss of ion $\langle d E / d x\rangle$. However, the actual energy loss scatters around the mean value and it is difficult to calculate it. Real detector cannot measure mean energy loss $\langle d E / d x\rangle$. It measures the energy $\Delta E$ deposited in layers of finite thickness $\Delta x$. For thin layers or low density materials, the energy loss distribution shows large fluctuations towards high losses, so called Landau tails. For high density materials, the energy loss distribution shows a more Gausian-like distribution. Form of distribution is important as energy distribution is often used for calibrating the detector for Rutherford Back Scattering (RBS) spectroscopy.

From a practical point of view, it is significant that uncertainty in the energy determination of the ion in the material volume can be easily determined from the straggling data. The low and high energy limits of the ion energy in the sample volume can be calculated for these data. Subsequently, the procedure of the ion energy uncertainty determination is shown in Fig.5.

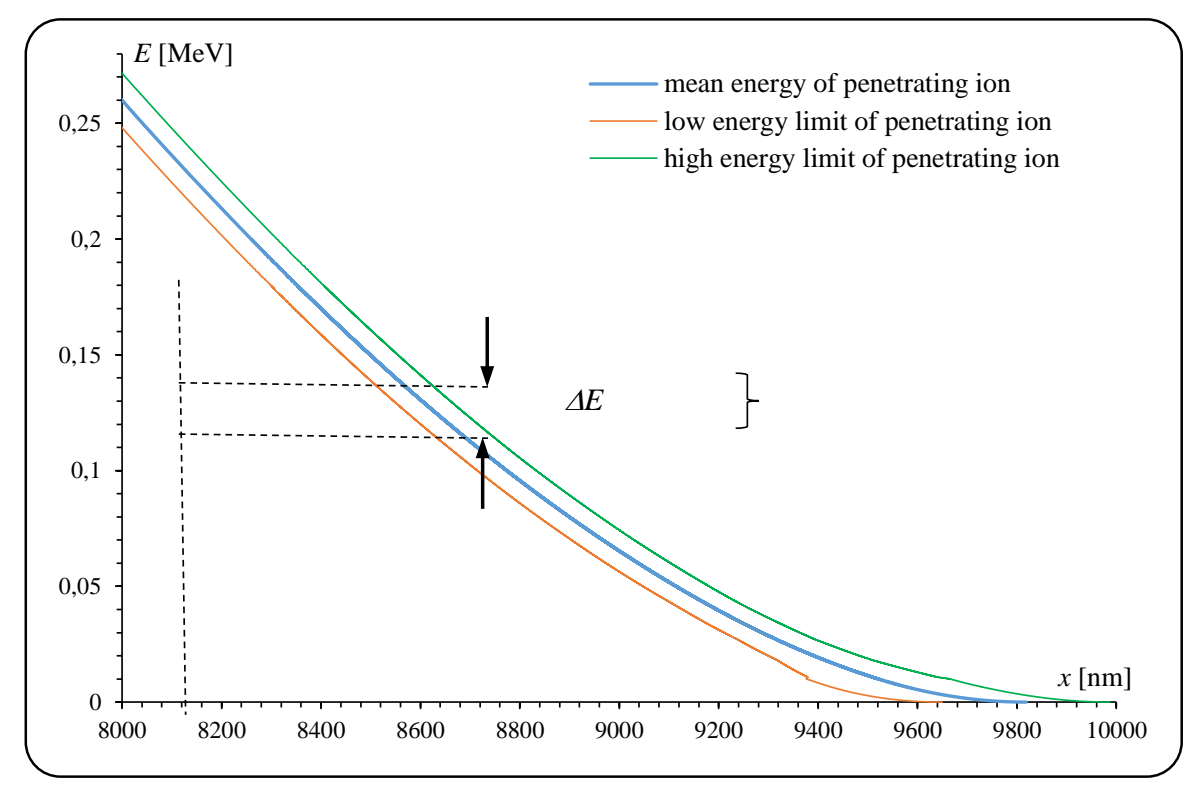

Fig. 5 Calculated energy decrease of $2.2 \mathrm{MeV} \mathrm{He}^{2+}$ ion penetrating in the kapton foil calculated taking into account straggling in the depth range from $8 \mathrm{~mm}$ up to $10 \mathrm{~mm}$. Uncertainty in the determination of ion energy $\Delta E$ at a given depth is illustrated.

\section{CONCLUSION}

The energy decrease of $2.2 \mathrm{MeV} \mathrm{He}^{2+}$ ion penetrating in the kapton foil using discrete data from SRIM database was treated analytically in this contribution. The major motivation in the research was to verify the applicability of a simple analytical algorithm for quantitative evaluation of the kapton foil depth. Results are applicable in a suitable processing of the experimental Elastic Recoil Data Analysis (ERDA) signal measured using the kapton foil.

The quantitative evaluation of the foil depth requires the conversion of the measured energy of ions versus the distance travelled by the ion in the material. Basically, this conversion procedure comprises the evaluation of the energy of ion after traveling a distance $x$ in the material structure using discrete stopping power data from the SRIM database. Data processing formulas were presented, starting with a very simple approximation of the stopping power dependence from the energy using the linear interpolation the SRIM data. The basic mathematical procedures for the quantitative depth evaluation were stated, and outputs as well 
as the processed data points were illustrated for the kapton. It can be assumed, that possible errors occur due to straggling.

We analytically solved the problem that is usually investigated using statistical algorithms in order to simplify and accelerate data processing. One can easily understand that there is a correlation between the energy of ion detected after scattering and the depth of the scattering centre if the energy of the ion penetrating into material changes. This correlation was mathematically demonstrated in our contribution.

\section{Acknowledgement}

This research output was supported by the Research and Development Operational Programme under the project of "University Scientific Park Campus MTF STU - CAMBO" ITMS: 26220220179.

\section{References:}

1. F. BLOCH, Z. 1933. Phys., 81, 363.

2. H. BETHE, Z. 1932. Phys., 76, 293.

3. H. BETHE und J. ASHKIN. 1953. Experimental Nuclear Physics. New York: ed. E. Segré, J. Wiley, p. 253.

4. U. FANO, CHR. 1964. Studies in Penetration of Charged Particles in Matter, Nucl. Sci. Rpt., 39, U. S. National Academy of Sciences, Washington, 1-338.

5. J. F. ZIEGLER. 1977. Helium Stopping Powers and Ranges in All Elemental Matter. New York: Pergamon.

6. P. SIGMUND. 2006. Particle Penetration and Radiation Effects. Springer Series in Solid State Sciences, 151. Berlin Heidelberg: Springer-Verlag.

7. J. LINDHARD, V. NIELSEN, M. SCHARFF. 1968. Mater. Fys. Medd. Dan. Vid. Selsk. 36, No. 10.

8. J. LINDHARD, M. SCHARFF, H.E. SCHIØTT. 1963. Mater. Fys. Medd. Dan. Vid. Selsk. 33, No. 14.

9. J. LINDHARD et al. 1963. Range concepts and heavy ion ranges. Mat. Fys. Medd. Dan. Vid. Selsk., Vol. 33, pp. 1-39.

10. S. FURUKAWA et al. 1972. Theoretical considerations on lateral spread of implanted ions. Jpn J. Appl. Phys., 11(2), pp. 134-142.

11. P.C.ZALM, P. BAILEY, M.A. READING, A.K. ROSSALL, J.A. VAN DEN BERG. Quantitative Considerations in Medium Energy Ion Scattering Depth Profiling Analysis of nanolayers. Nucl. Instr. and Meth. B, 387, (2016), 77-85. (1998) 1157.

12. J.F.ZIEGLER, J.P. BIERSACK, U. LITTMARK. 1985. The Stopping and Range of Ions in Matter. New York: Pergamon.

13. J.F.ZIEGLER, J.P. BIERSACK, U. LITTMARK. 1985. The Stopping and Range of Ions in Matter. New York: Pergamon.

14. K.S.C. TRAUTMANN, R. NEUMANN. 2003. Electronic Excitations and Heavy-Ion-Induced Processes in Ionic Crystals. Nucl.Instr.Meth.Phys.Res B 209, 73.

15. L.G.GLAZOV. 2002. Frozen-Charge Stopping of Ions in the Bethe Regime. Nucl.Instr.Meth.Phys.Res B 195, 118.

16. G. MOLIERE. 1947. Theorie der Streuung schneller geladener Teilchen I; Einzelstreuungam abbgeschirmten Coulomb-Feld, Z. f. Naturforsch, A2 133.

17. Interaction of Charged Particles with Solids and Surfaces. Edited by A. Gras-Marti, H.M. Urbassek, N. Arista, F. Flores, (Plenum Press, New York, 1991). 